Proceedings of Magneto-Optical Recording Intemational Symposium 97. J. Magn. Soc. Jpn., Vol. 22 Supplement No. S2 (1998), pp. 105-108 C1997 by The Magnetics Society of Japan

\title{
TEMPERATURE DEPENDENCE OF THE AMORPHOUS SmTbFeCo MAGNETO-OPTICAL FILMS
}

\author{
Z. Y. Lee Z. Q. Lu Y. K. Zheng J. J. Qiu K. Wang R. Xiong S. F. Yang Z. Q. Hu \\ Dept. of Solid State Electronics, Huazhong University of Sci, and Technol. Wuhan, 430074. P. R. China
}

\begin{abstract}
A mean- field theory is developed to research the temperature dependence of the amorphous SmTbFeCo thin films. It is shown that with available $\mathrm{Sm}$ substitution of $\mathrm{Tb}$, the composition temperature $T_{\text {comp }}$ decreases, Curie temperature $T_{c}$ remains unchanged, Kerr angle becomes larger, the saturation magnetization $M s$ at room temperature increases. Therefore, LRE-HRE-TM amorphous films can be used as the high density magneto-optical media in a wide range of temperature. However, the Sm substitution of TM does not improve the magneto-optical performance. KEYWORDS TEMPERATURE DEPENDENCE, MAGNETO-OPTICAL FILMS, MEAN-FIELD THEORY.
\end{abstract}

The heavy rare earth - transition metal ( HRE-TM ) amorphous alloys are now commercially used as magneto-optical (MO) media [1-2]. Their MO Kerr angles are about $0.3^{\circ}$ at wavelength region of 780 $830 \mathrm{~nm}$, but decrease with decreasing wavelength. For high density MO recording, it is essential to develop materials which have a high MO performance at short wavelengths. The light rare earth (LRE) can exhibit a large MO Kerr angle in amorphous alloys with TM[35]. However, the recording properties of these alloys are not suitable as MO media. To make a MO storage medium which takes advantage of these improved properties, it is necessary to incorporate the light rare earth into HRE-TM alloys. Until now it has not clear if the addition of LRE into HRE-TM alloys enhances MO performance $[8-9]$. The present study is motivated by the desire to understand the magnetic and magneto-optical properties in pseudo-temary alloy films of $\mathrm{SmTbFeCo}$ and the behavior of magnetization versus temperature.

\section{MEAN-FIELD THEORY}

The mean-field theory becomes cumbersome whien ternary and quatemary samples are treated since the parameters are numerous and adjustment is rather arbitrary. As an alternative, we consider modeling quatemary alloys of RE-TM with three sub-networks. The first sub-network consists of HRE atoms $x_{1}=x_{\text {FiR }}$. The second sub-network consists of LRE atoms $x_{2}=x_{1 R R: \text { : }}$ The third sub-network consists of TM atoms $x_{3}=x_{1 M}$.

The localized mean-field theory is based on the Heisenberg Hamiltonian. The energy of interaction between atoms $i$ and $j$ with average angular momenta $s_{i}$ and $s_{j}$ is given by $[5-6]$ :

$$
e_{i j}=-2 J_{i j} s_{i} s_{i}
$$

where $s_{i}$ and $s_{j}$ are the projections of angular momenta along the axis of the anisotropy and $J_{i j}$ is the exchange interaction energy. The total exchange energy of $i$ arising from interactions with its nearest neighbors in our triple-magnetic-subnetwork model is thus given by

$$
E_{i}=-Z_{i} s_{i} \sum_{j=1}^{3} x_{j} J_{i j}\left\langle s_{j}>\right.
$$

where $Z_{i}$ is coordination number, $x_{j}$ is atomic percentage of the jth species in the compound, then

$$
\begin{gathered}
<s_{i}>=\left(S_{i}+\frac{1}{2}\right) \operatorname{coth}\left[\frac{Z_{i}\left(2 S_{i}+1\right) \sum_{j=1}^{j} x_{j} J_{i j}<s_{j}>}{2 k T}\right] \\
-\frac{1}{2} \operatorname{coth}\left[\frac{Z \sum_{j=1}^{3} x_{j} J_{i j}<s_{j}>}{2 k T}\right]
\end{gathered}
$$

The subnetwork magnetization are then obtained from the relation

$$
M_{i}=N x_{i} \mu_{B} g_{i}<s_{i}>
$$

$\mu^{\mu} B$ is the Bohr magneton, $g_{i}$ is gyromagnetic factor, and $N$ is the total number of atoms( ions ) in unit volume. Since amorphous materials are usually less dense than their crystalline counterparts, we assume that only $95 \%$ percent of the space is filled in the amorphous state. the total number of atoms per unit volume $N$ is thus given by

$$
N=\frac{0.95}{\sum_{i=1}^{n} 4 \pi r_{i}^{3}{ }^{3}}
$$

$r_{i}$ is the atomic radius.

\section{EXPERIMENTS}

SmTbFeCo films with perpendicular magnetic anisotropy have been deposited by $\mathrm{r} f$. magnetron 
sputtering from a mosaic target onto glass substrate. The sputtering conditions are as follows: background pressure $1.07 \times 10^{-4} \mathrm{~Pa}$; Ar pressure $0.4 \sim 1.0 \mathrm{~Pa}$; the sputtering power $300 \mathrm{~W}$; substrate temperature

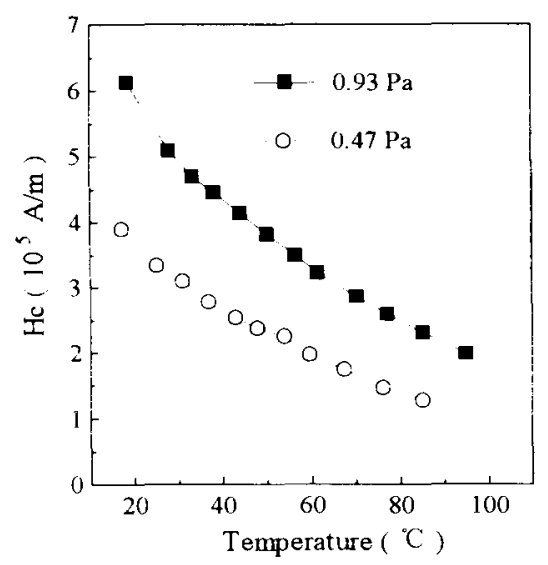

Fig. 1 The temperature dependence of the coercivity of amorphous SmTbFeCo films

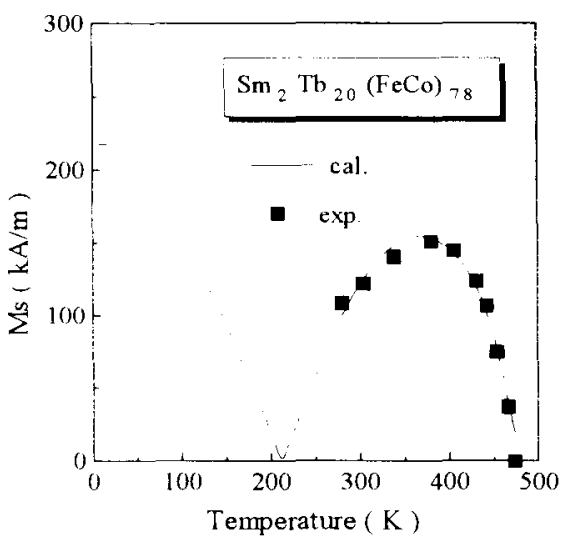

Fig.2 Temperature dependence of the saturation magnetization of amorphous SmTbFeCo films

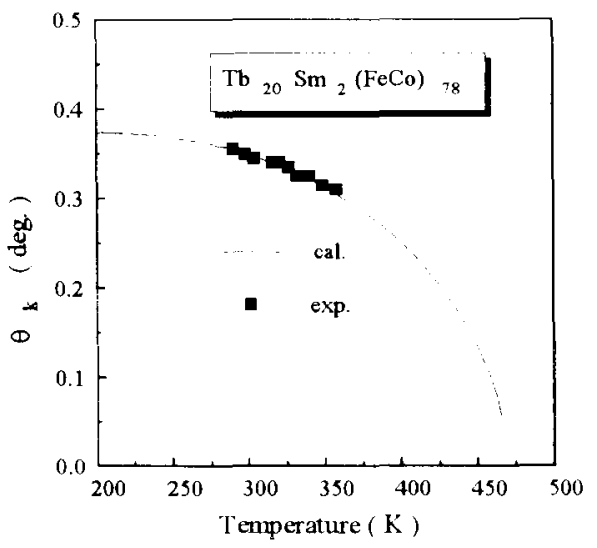

Fig. 3 Temperature dependence of the Kerr rotation angle of amorphous SmTbFeCo films

$80 \sim 100{ }^{\circ} \mathrm{C}$. We have also deposited AlN film using r.f. reactive sputtering for $\mathrm{RE}$ metal selective oxidation protection. The layer configuration is $\mathrm{AlN} / \mathrm{SmTbFeCo}[9]$. The coercivity $\mathrm{Hc}$ and Kerr angle $\theta_{k}$ are measured from Kerr hysteresis, and the saturation magnetization $M s$ by using a vibrating sample magnetometer.

The temperature dependencies of coercivity $H c$, saturation magnetization $M s$ and Kerr angle $\theta_{k}$ are shown in Fig. 1, Fig. 2 and Fig. 3 respectively. As shown in Fig. 1, $H c$ exhibits a rise with film fabricated under high pressure. Microvoids and columnar structure may act as pinning points in magnetic domain walls, increasing $H c$. It is also shown that at the same sputtering conditions, the coercivity of SmTbFeCo film with available $\mathrm{Sm}$ substitution of $\mathrm{Tb}$ is larger than that of $\mathrm{TbFeCo}$ film, the coercivity versus temperature becomes more slowly, and its Kerr angle can be $0.33^{\circ}$, which is larger than that of $\mathrm{TbFeCo}$.

Table 1 The parameters of SmTbFeCo amorphous films used in calculation of Ms-T curve

\begin{tabular}{|c|c|c|c|c|}
\hline & & $\mathrm{Tb}$ & $\mathrm{Sm}$ & $\mathrm{FeCo}$ \\
\hline atomic radius & $\mathrm{r}(\mathrm{nm})$ & 22.4 & 22.4 & 16.5 \\
\hline gyromagnetic factor & $\mathrm{g}$ & 1.5 & $2 / 7$ & 2.2 \\
\hline angular quantum number & $\mathrm{S}$ & 6 & adjustable & \\
\hline coordination number & $Z$ & & 12 & \\
\hline
\end{tabular}

Table 2 The fitting parameters of the spins and exchange integrals of SmTbFeCo films

\begin{tabular}{cccccccc}
\hline$S_{\mathrm{Sm}}$ & $S_{\mathrm{TM}}$ & $J_{\mathrm{TbTb}}$ & $J_{\mathrm{TbSn}}$ & $J_{\mathrm{TbTM}}$ & $J_{\text {SmSm }}$ & $J_{\text {SimTM }}$ & $J_{\mathrm{TM} \text { IM }}$ \\
\hline 2.51 & 0.67 & 3.67 & -3.10 & -2.63 & 2.91 & 5.18 & 90.38 \\
\hline
\end{tabular}




\section{RESULTS AND DISCUSSION}

We use the above mean-field theory to verify the experimentally observed behavior of saturation magnetization versus temperature in SmTbFeCo alloys. the parameters are listed in table 1 . The value of the parameters $S_{\mathrm{Sm}}, S_{\mathrm{TM}}, J_{\mathrm{SmSm}}, J_{\mathrm{ThSm}}, J_{\mathrm{SmTM}}$ and $J_{\mathrm{TMTM}}$ are determined to fit the experimental data on the temperature dependencies of $M s$ shown in Fig. 2. The obtained value of the parameters are listed in table 2 . Using the parameter values obtained above, the calculated values fitted well with the experimental values.

The temperature dependencies of $M s$ of $\mathrm{SmTbFeCo}$ at a constant TM content are shown in Fig. 4. The more $\mathrm{Tb}$ are replaced by $\mathrm{Sm}$, the lower the compensation temperature, the larger the saturation magnetization $M s$ at the room temperature, and the Curie temperature remains nearly unchanged. It is also found that with available $\mathrm{Sm}$ substitution of $\mathrm{Tb}$, the saturation magnetization $M s$ versus temperature changes more slowly. Thus, SmTbFeCo amorphous films can be used as the high density magneto-optical recording media in a wide range of temperature.

The temperature dependencies of $M s$ of $\mathrm{SmTbFeCo}$ at a constant $\mathrm{Tb}$ content are shown in Fig. 5. With TM replaced by $S \mathrm{~m}$, the compensation temperature $T_{\text {comp }}$ becomes higher, the Curie temperature $T_{\mathrm{c}}$ tends to lower, and the saturation magnetization $M s$ at the room temperature decreases.

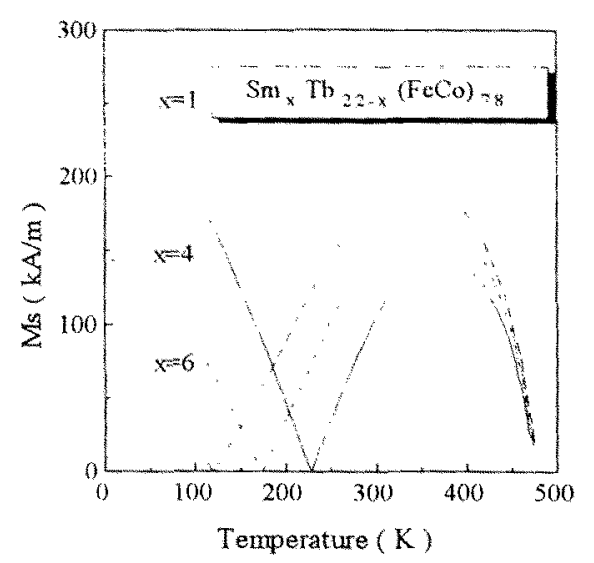

Fig.4 Temperature dependence of the saturation magnetization $M s^{\prime}$ for $S m T b F e C o$ films at a constant TM content

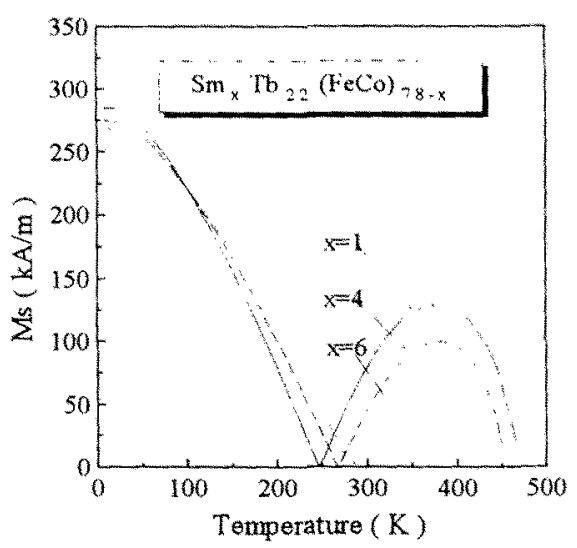

Fig. 5 Temperature dependence of the saturation magnetization $M s$ for SmTbFeCo films at a constant $\mathrm{Tb}$ content

In the HRE-LRE-TM films, the Kerr effect is attributed to the sublattice magnetic moments. The Kerr rotation angle $\theta_{k}$ of HRE-LRE-TM films will be represented by

$$
\begin{aligned}
\theta_{*} & =K(T M) M s_{T M}+K(L R E) M s_{L R E} \\
& -K(H R E) M s_{H R E}
\end{aligned}
$$

where value of the coefficients $K(\mathrm{TM}) 、 K(\mathrm{LRE})$ and $K$ (HRE) are determined such that eq $(7)$ fitted the experimental data. the samples are shown in Fig. 3 . the obtained values of the coefficients $K(\mathrm{TM}), K(\mathrm{Sm})$ and $K(\mathrm{~Tb})$ are $9.45 \times 10^{-7}, 4.05 \times 10^{-7}$ and $1.03 \times 10^{-7}$ $\mathrm{deg} \cdot \mathrm{m} / \mathrm{A}$ respectively

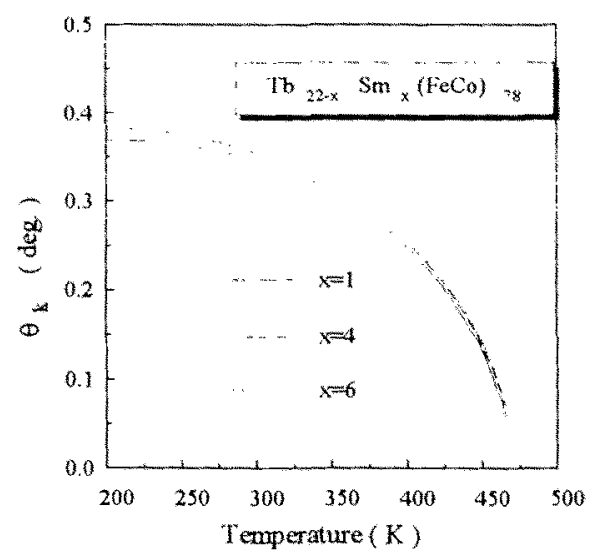

Fig. 6 Temperature dependence of the Kerr rotation angle $\theta_{k}$ of amorphous SmTbFeCo films at a constant TM content 


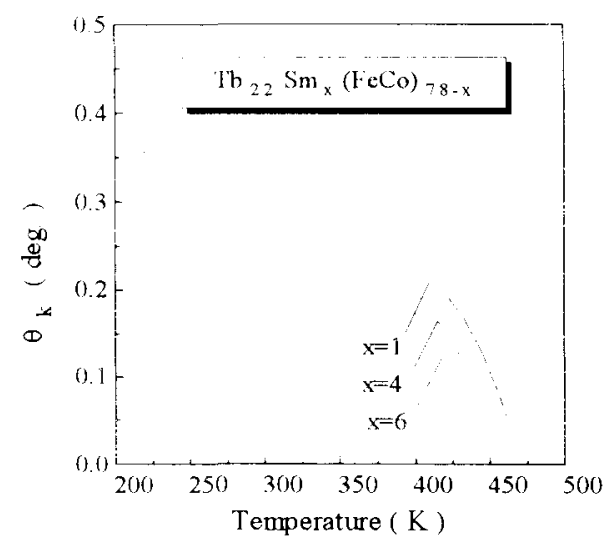

Fig. 7 Temperature dependence of the Kerr rotation angle $O_{k}$ of amorphous $\mathrm{SmTbFeCo}$ films at a constant $\mathrm{Tb}$ content

The temperature dependencies of the Kerr rotation angles $\theta_{k}$ of SmTbFeCo films at constant TM content are shown in $\mathrm{F} 1 \mathrm{~g}$. 6. With $\mathrm{Sm}$ substitution of $\mathrm{Tb}$, the Kerr rotation angle $\theta_{k}$ increases. Therefore, the available $\mathrm{Sm}$ substitution of $\mathrm{Tb}$ can enhance the magneto-optical effect. The temperature dependencies of the Kerr rotation angles $\theta_{k}$ of SmTbFeCo films at constant $\mathrm{Tb}$ content are shown in Fig. 7. With $\mathrm{Sm}$ substitution of TM, the Kerr rotation angle $\theta_{k}$ decreases Thus. Sm substitution of TM does not enhance the magneto-optical effect. Therefore, we can explain why the addition of LRE into TbFeCo films enhances MO performance at same time or not at other time.

\section{CONCLUSION}

A mean-field model is applied to $\mathrm{SmTbFeCo}$ films and to explain the behaviors of the saturation magnetization and Kerr rotation angle versus temperature It is shown that the available $\mathrm{Sm}$ substitution of $\mathrm{Tb}$ can cause the composition temperature $T_{\text {comp }}$ to decrease, the Curie temperature $T_{\mathrm{c}}$ remains unchanged, the Kerr rotation angle becomes larger, and the saturation magnetization $M S$ at room temperature increases. Therefore, LRE-HRE-TM amorphous films can be used as the high density magneto-optical media in a wide range of temperature. On the contrary, with $\mathrm{Sm}$ substitution of $\mathrm{TM}$, the composition temperature $T_{\text {comp increases, Curie }}$ temperature $T_{\mathrm{C}}$ decreases, the Kerr rotation angle becomes small, and the saturation magnetization $M s$ at room temperature decreases. Therefore, it does not improve the magneto-optical performance. Thus, we can explain whether the addition of LRE into HRE-TM alloys enhances MO performance.

\section{REFERENCES}

[1] Z. Y. Lee. X, S. Miao, P. Zhu, et al. J Magn Magn Mater, 115 ( 1 ): 44-54( 1992 )

[2] R. Carey, D. M. Newman, B. W. J. Thomas. J Phys D: Appl Phys, 28: 2207-2227( 1995 )

[3] Z. Y. Lee, X. S. Miao, P. Zhu, et al .J Magn Soc Jpn, 15 ( 8 ): 421-424 ( 1991 )

[4] Z. Q. Lu, Z. Y. Lee, Y. K. Zheng, et al . SPIE, 2890: 20-25 ( 1996$)$

[5] M. Mansuripur, and M. F. Ruane. IEEE Trans Magn , 22(1): 33 ( 1986 )

[6] S. Honda, and M. Yoshiyama. Jpn J Appl Phys .27(9): 1687-1692 ( 1988 )

[7] Z. Q. Lu, Z. Y. Lee, Y. K. Zheng, et al. Chin Phys Lett, 14(2): 131-133 ( 1997 )

[8] M. Nakada, H. Yokota, S. Yumoto. IEEE Trans Magn ,25(5): 3767-3769 ( 1989 )

[9] C. B. Peng, W. M. Kim, B. K. Cheong, et al . J Appl Phys, 80(8): 4498-4502 ( 1996 ) 